\title{
Coulomb Energy Systematics *
}

\author{
JOACHIM JÄNECKE \\ Department of Physics, The University of Michigan, Ann Arbor, Michigan
}

Received June 21, 1966

A new type of plot for the Coulomb energy differences between isobaric analogue states of neighboring isobaric nuclei with $T_{z}=T-1$ and $T_{z}=T$ is introduced. The available experimental data when displayed in this form reveal nuclear structure effects.

\section{Introduction}

Many new experimental data on Coulomb energy differences in light, intermediate and heavy nuclei are now available. The increasing number and accuracy of these data makes it possible to refine their earlier description and interpretation in terms of a homogeneous spherical charge distribution. Pronounced and apparently unsystematic departures from such a description have been established by HARCHOL et al. ${ }^{1}$ and can be seen in a plot of $\Delta E_{\text {Coul }} A^{\frac{1}{3}} / Z$ as a function of $Z / A^{\frac{1}{3}}$. The systematics of these departures, which are presumably due to nuclear structure effects, should become more evident when $\Delta E_{\text {Coul }}$ or related quantities are considered as a function of $t w o$ variables, for instance $Z$ and $N$ or $A$ and $T_{z}$. The purpose of the present paper is to introduce and to discuss a new type of plot for the Coulomb energy differences between the isobaric analogue states of neighboring isobaric nuclei with $T_{z}=T-1$ and $T_{z}=T$ as a function of $A$ and with the isobaric spin $T$ as a parameter.

\section{General Considerations}

In first order perturbation theory the $T_{z}$ dependence of the Coulomb energies can be given as ${ }^{2}$

$$
\begin{aligned}
& E_{\mathrm{Coul}}\left(A, T, T_{z}\right) \\
& \quad=E_{\text {Coul }}^{(0)}(A, T)-T_{z} E_{\text {Coul }}^{(1)}(A, T)+\left[3 T_{z}^{2}-T(T+1)\right] E_{\text {Coul }}^{(2)}(A, T) .
\end{aligned}
$$

The quantities of primary interest are the vector and tensor Coulomb energies $E_{\text {Coul }}^{(1)}$ and $E_{\text {Coul }}^{(2)}$ which depend on $A, T$ and the detailed pro-

* This work was supported in part by the U.S. Atomic Energy Commission.

${ }^{1}$ Harchol, M., S. Cochavi, A.A. JAfFe, and Ch. Drory: Nuclear Phys. 79, 165 (1966).

2 Wigner, E.P.: Proc. Robert A. Welch Foundation Conf. on Chemical Research, Vol. I, Houston/Texas 1957, p. 88. - WeInBerg, S., and S.B. Treiman: Phys. Rev. 116, 465 (1959). - WILKINSON, D.H.: Phys. Letters 11, 243 (1964); 12, 348 (1964); Phys. Rev. Letters 13, 571 (1964). 
perties of the system. The $A$-dependence of the experimental vector and tensor Coulomb energies has been studied ${ }^{3,4}$ in the light nuclei up to $A=43$. In the intermediate and heavy nuclei many isobaric analogue states with $T=T_{z}+1$ have been observed. "Double analogue" states with $T=T_{z}+2$ have also been observed ${ }^{5}$ in two heavier nuclei. The $A$ and $T$-dependence of $E_{\mathrm{Cou} 1}^{(1)}$ and $E_{\mathrm{Cou1}}^{(2)}$, however, can only be studied in this mass region if more data on isobaric analogue states with $T=T_{z}+2$ and the related Coulomb energy differences become available. Then, $E_{\text {Coul }}^{(2)}$ and $E_{\text {Coul }}^{(1)}$ can be obtained from

$$
\begin{aligned}
& \begin{aligned}
& E_{\text {Coul }}^{(1)}(A, T) \\
&=\left(T-\frac{1}{2}\right) \Delta E_{\text {Coul }}(A, T, T-2 \mid T-1)-\left(T-\frac{3}{2}\right) \Delta E_{\text {Coul }}(A, T, T-1 \mid T) \\
&= \Delta E_{\text {Coul }}(A, T, T-1 \mid T)+ \\
& \quad+\left(T-\frac{1}{2}\right)\left\{\Delta E_{\text {Coul }}(A, T, T-2 \mid T-1)-\Delta E_{\text {Coul }}(A, T, T-1 \mid T)\right\}
\end{aligned} \\
& \text { and } \quad \\
& \qquad E_{\text {Coul }}^{(2)}(A, T)=\frac{1}{6}\left\{\Delta E_{\text {Coul }}(A, T, T-2 \mid T-1)-\Delta E_{\text {Coul }}(A, T, T-1 \mid T)\right\} .
\end{aligned}
$$

Here,

$$
\Delta E_{\mathrm{Coul}}\left(A, T, T_{z} \mid T_{z}^{\prime}\right)=E_{\mathrm{Coul}}\left(A, T, T_{z}\right)-E_{\mathrm{Coul}}\left(A, T, T_{z}^{\prime}\right) .
$$

In the following we will be concerned with the Coulomb energy differences only, that is with the following combination of vector and tensor Coulomb energies

$$
\Delta E_{\text {Coul }}(A, T, T-1 \mid T)=E_{\text {Coul }}^{(1)}(A, T)-3(2 T-1) E_{\text {Coul }}^{(2)}(A, T) .
$$

In the approximation of a homogeneously charged sphere $\Delta E_{\text {Coul }}$ essentially becomes a linear function of $Z / A^{\frac{1}{3}}$. It has been shown ${ }^{6-8}$ that the gross structure of the experimental data is well described by this equation. A more detailed examination of the data, however, shows noticeable fluctuations in the dependence of $\Delta E_{\mathrm{Coul}}$ on $Z / A^{\frac{1}{3}}$.

CARLSON and TALMI ${ }^{9}$ have derived a Coulomb energy formula assuming that the states under consideration have lowest seniority and that

3 JÄNECKE, J.: Phys. Rev. 147, 735 (1966).

4 JÄNECKE, J.:Proc. Conference on Isobaric Spin in Nuclear Physics. Tallahassee 1966, p. 60.

${ }^{5}$ Batty, C.J., E. Friedman, P.C. Rowe, and J.B. Hunt: Phys. Letters 19, 33 (1965).

6 Anderson, J.D., C. Wong, and J.W. McClure: Phys. Rev. 138, B 615 (1965).

7 Sherr, R., B.F. Bayman, E. Rost, M.E. Rickey, and C.G. Hoot: Phys. Rev. 139, B 1272 (1965).

${ }^{8}$ Batty, C.J., R. S. GILMORE, and G.H. StAfFord: Nuclear Phys. 75, 599 (1966).

9 CArlson, B.C., and I. Talmi: Phys. Rev. 96, 436 (1954). - DE-Shaltr, A., and I. TALMI: Nuclear shell structure, p. 345. New York and London: Academic Press 1963. 
there are only $Z^{\prime}$ protons in a $j^{n}$ configuration outside a closed shell. They obtained

$$
E_{\mathrm{Coul}}=Z^{\prime} C+\frac{1}{2} Z^{\prime}\left(Z^{\prime}-1\right) a+\left[\frac{Z^{\prime}}{2}\right] b
$$

Eq. (5) probably holds also approximately for other cases, i.e. when the configurations are more complicated than $j^{n}$, or when one has protons and neutrons outside the core. The pairing term, however, has to be modified for the latter case to comply with Eq. (1). Using a modified pairing term ${ }^{3}$ one obtains from Eq. (5)

$$
\begin{gathered}
\Delta E_{\text {Coul }}(A, T, T-1 \mid T)=E_{0} A-E_{1} T+E_{2}+\delta(A, T) \frac{E_{3}}{2 T} \\
\delta(A, T)=\frac{1}{2}\left[1-(1-1)^{\frac{A}{2}-T}\right]= \begin{cases}0 & \text { for } \frac{A}{2}-T \text { even } \\
1 & \text { for } \frac{A}{2}-T \text { odd }\end{cases}
\end{gathered}
$$

with $E_{1}=2 E_{0}$. The energy coefficient $E_{i}$ are related to the electrostatic interaction between protons.

The pairing term in Eq. (6) predicts oscillations in the $A$-dependence of $\Delta E_{\text {Coul }}$ for fixed $T$. The amplitude of these super-imposed oscillations should decrease with increasing $T$. The pairing term should therefore be of significance only for the light nuclei. The marked difference in the observed amplitudes (factor of 2) for $T=\frac{1}{2}$ and $T=1$ has been noticed before $^{10}$ but was not explained. From the $A$ - and $T$-dependence of the numerical coefficient $\delta(A, T)$ in the last term of Eq. (6) it follows also that for both even- $A$ and odd- $A$ nuclei ( $T$ is integer and half-integer, respectively) there should be well defined phase relations between the above oscillations for different $T$. Such effects have also been noticed ${ }^{7}$ recently in a study of second Coulomb energy differences.

All energy coefficients $E_{i}$ are related to expectation values of $1 / R$. Due to the change in radius they should therefore exhibit an $A$-dependence to the form $1 / A^{\frac{1}{3}}$. Eq. (6) can then be rewritten as

$$
\Delta E_{\mathrm{Coul}}(A, T, T-1 \mid T)=\frac{1}{A^{\frac{1}{3}}}\left\{e_{0} A-e_{1} T+e_{2}+\delta(A, T) \frac{e_{3}}{2 T}\right\} .
$$

The coefficients $e_{i}$ should be constant for a given type of configuration but they may vary at shell crossings. Since Eq. (7) is still only an approximation one might expect that in reality even within a given type of

10 J̈̈NECKE, J.: Z. Physik 160, 171 (1960). 
configurations the quantities $e_{i}$ become functions (hopefully weak) of $T$ and/or $A$ and that $e_{1} \approx 2 e_{0}$ only. This expectation is strongly confirmed both theoretically and experimentally.

Based on the seniority scheme $\mathrm{HECHT}^{11}$ has calculated expressions for the Coulomb energies of states with $j^{n}$ configurations. It follows from his equations that the energy coefficients $e_{i}$ are not exactly constant but become weak functions of $T$ and $A$. The coefficients $e_{i}$ which are related to the electrostatic interaction between protons and the electromagnetic interaction between nucleons can, in principle at least, be calculated.

It has been shown experimentally ${ }^{12}$ that in some cases the Coulomb energy differences $\Delta E_{\text {Coul }}$ between the isobaric analogue states of a sequence of pairs of isotopes do not vary with $A^{-\frac{1}{3}}$. Considering Eq. (7) such behavior is possible only if at least one of the following statements holds: (i) $e_{0}, e_{1}$ or $e_{2}$ are functions of $T$ and/or $A$, (ii) $e_{1} \neq 2 e_{0}$, (iii) $e_{3} \neq 0$ [relevant only for the light nuclei and for the case where $\delta(A, T)=1$ ].

\section{Graphical Display of the}

\section{Coulomb Energy Differences and Discussion}

Fig. 1 shows the variations with $A$ of the reduced Coulomb energy differences ${ }^{\star} \Delta E_{\text {Coul }}^{\text {(red) }}(A, T, T-1 \mid T)=\Delta E_{\text {Coul }}(A, T, T-1 \mid T)-\delta(A, T) \frac{E_{3}}{2 T}$. The isobaric spin $T$ is used as a parameter. The subtraction of the pairing energy term with $E_{3}=120 \mathrm{keV}$ virtually eliminates all oscillations. The experimental data for $T=\frac{1}{2}$ and $T=1$ exhibit the well-known discontinuities at the shell closures. Straight lines were drawn through the points to display these effects. All experimental points for $T>1$ were connected by straight lines and no effort was made to display shell effects.

Such effects can be demonstrated better in two different kind of plots. The slope $E_{0}$ of the various lines in Fig. 1 decreases with increasing $A$. Also, the energies for a given and constant $\bar{Z}$ decrease with increasing $A$ (see for instance the 8 points between $A=113, T=\frac{13}{2}$ and $A=125, T=\frac{25}{2}$ ). Both effects are to be expected. They are due to the

* Most of the data were taken from the compilations in refs. 1, 3, 6, and 7. Some additional data were also included (Refs. 8, 12, and 13).

${ }^{11}$ Hecht, K.T.: Proc. Conference on Isobaric Spin in Nuclear Physics, Tallahassee 1966; private communication.

12 Sherr, R., A.G. Blatr, and D.D. Armstrong: Phys. Letters 20, 392 (1966).

13 Lauritsen, T., B. LynCH, and G. Griffrths: Bull. Am. Phys. Soc. 8, 597 (1963). - Cerny, J., R.H. PeHL, and G.T. Garvey: Phys. Letters 12, 234 (1964). KIM, H.J., C. M. Jones, H.B. Willard, and J. K. Blair: Bull. Am. Phys. Soc. 11, 100 (1966). - Hamburger, E.W., J. Kě̃eméneK, B.L. Cohen, and J. Moorehead: Bull. Am. Phys. Soc. 11, 119 (1966). 
increase in radius with increasing $A$. Therefore, as one can see from Eq. (7), it appears reasonable to plot the quantity $A^{\frac{1}{3}} \Delta E_{\text {Coul }}^{(\text {red })}$ as a function of $A$. Fig. 2 shows that the slopes $e_{0}$ of the various lines indeed become essentially constant and independent of $A$ and $T$. This statement cannot

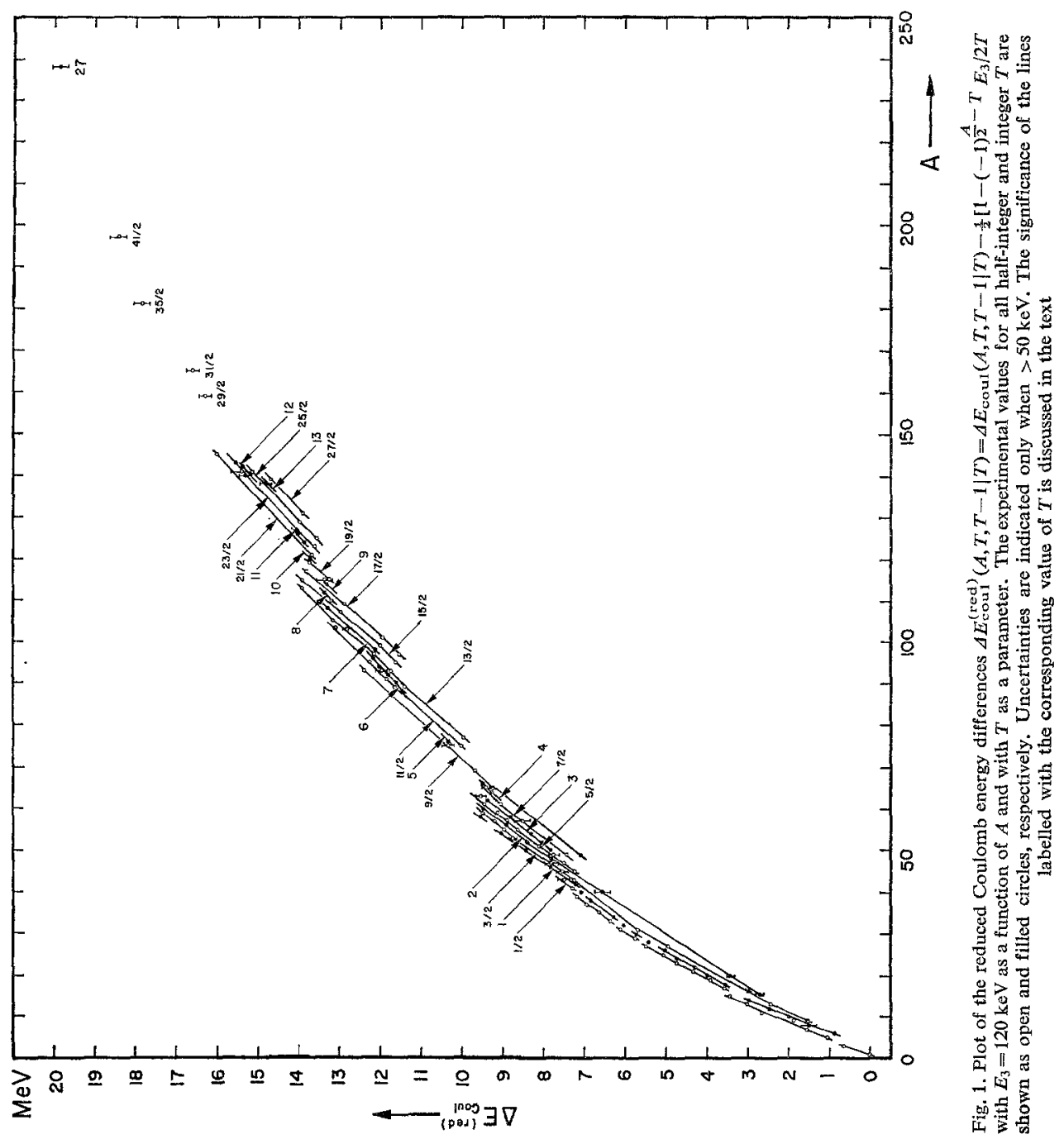

be exactly true, though. In several cases the quantity $A^{\frac{1}{3}} \Delta E_{\text {Coul }}^{\text {(red }}$ is not a function of $\bar{Z}$ only (see for instance $\bar{Z}=20.5,21.5,22.5,40.5,42.5$, $50.5 ; A^{\frac{1}{3}} \Delta E_{\mathrm{Cou} 1}^{(\mathrm{red})} \approx 25,27,28,53,56,68 \mathrm{MeV}$ ) but depends still on $N$, that is on $T$ and/or $A$. The trend seems to be that for a sequence of $33^{*}$ 
pairs of isotopes the Coulomb energy differences $\Delta E_{\text {Coul }}^{(\text {red })}$ decrease slower than $A^{-\frac{1}{3}}$ at the beginning of a new shell or a new type of configuration $[\bar{Z}>(8), 20,50]$, they follow essentially a $A^{-\frac{1}{3}}$-dependence in the middle, and they decrease faster than $A^{-\frac{1}{3}}$ at the end $[Z<(20), 50]$.

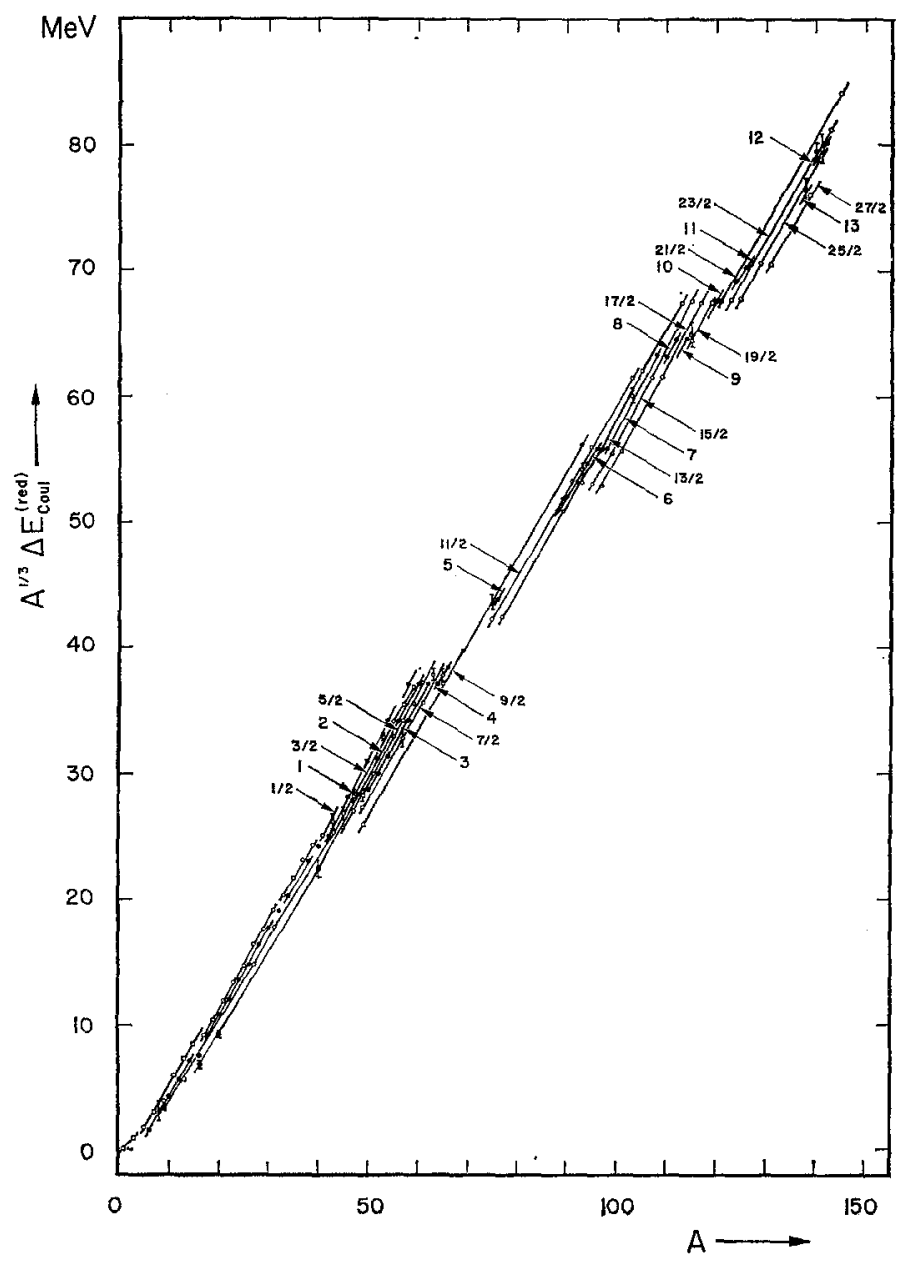

Fig. 2. Plot of the quantity $A^{\frac{1}{3}} \Delta E_{\text {coul }}^{\text {(red })}$ as a function of $A$

If one subtracts an $A$-dependent term of the form $\left\langle e_{0}\right\rangle_{\mathrm{av}} A$ from the quantities plotted in Fig. 2 one obtains a plot which is particularly suited to the study of fine structure effects. Fig. 3 shows a plot of the quantity $A^{\frac{1}{3}} \Delta E_{\text {Coul }}^{\text {(red }}-(0.722 \mathrm{MeV}) A$ as a function of $A$ and with $T$ as a parameter. The numerical coefficient for the subtracted term was taken 
from a best fit to the data in terms of a homogeneous charge distribution ${ }^{6}$. An analysis of this plot leads to the following findings.

For a homogeneous spherical charge distribution Fig. 3 should show continuous horizontal, parallel and equally spaced lines with a

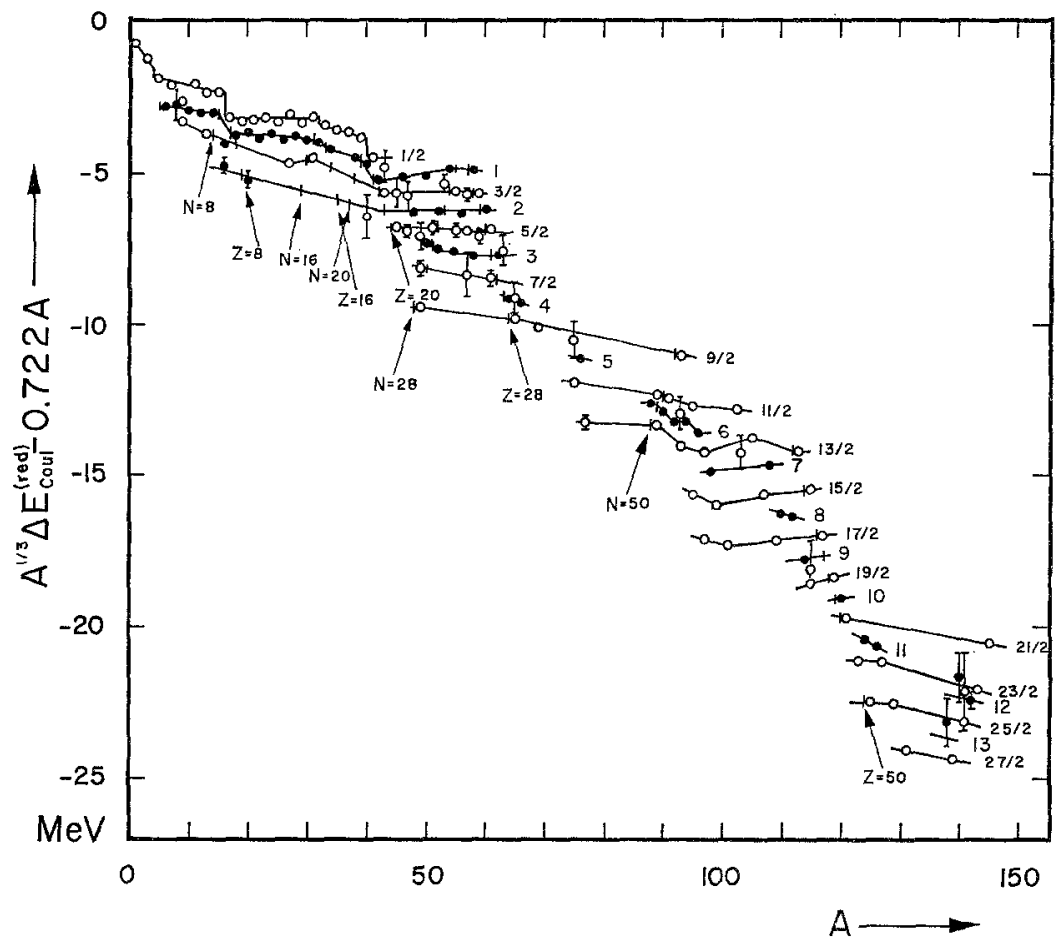

Fig. 3. Plot of the quantity $A^{\frac{1}{3}} \Delta E_{\text {coul }}^{\text {(red }}-(0.722 \mathrm{MeV}) A$ as a function of $A$

spacing of $0.722 \mathrm{MeV}$ between lines which differ in isobaric spin by $\frac{1}{2}$. This is not the case. The lines are not continuous and horizontal, but in a given region of $A$ they are at least approximately parallel and equally spaced.

Pronounced discontinuities occur at the major shell closures at $A=4,16$ and 40 for both $T=\frac{1}{2}$ and $T=1$. Less pronounced peaks can also be seen for $A=32$ and 56. Not enough data are available yet to definitely establish similar shell closure effects for $T>1$. The known points for $T=\frac{3}{2}$, however, strongly suggest such a behavior and there is no doubt that such effects will eventually show up at least for $T=\frac{3}{2}$ and $T=2$ in the light nuclei. For a given $T>\frac{1}{2}$ every magic nucleon number $n_{0}$ can lead to two discontinuities as a function of $A$, one for $N=n_{0}$ and the other for $Z=n_{0}$. Therefore, one should expect to observe one discontinuity above $A=2\left(n_{0}-T\right)$ and another one below $A=2\left(n_{0}+T\right)$. 
Note that the respective values of $A$ depend on $T$. The values of $A$ for which such discontinuities may appear were calculated for $n_{0}=8,16$, 20, 28 and 50. They are marked in Fig. 3 by small vertical lines and in addition indicated by arrows.

The point for $A=9, T=\frac{1}{2}$ is definitely too low due to a ThomasEhrman shift ${ }^{14}$ in $B^{9}$. Several other points with larger experimental errors also deviate appreciably from the expected values.

The slope of the various lines seems to increase with $j$. This implies that $e_{0}$ is $j$-dependent. Considering the $1 f_{\frac{7}{2}-}, 1 d_{\frac{5-}{2}}, 1 p_{\frac{3}{2}-}$ and $1 d_{\frac{3}{2}}$-shells, one has (at least for $T=\frac{1}{2}$ and $\left.T=1\right) e_{0}\left(j=\frac{7}{2}\right)>0.722 \mathrm{MeV}, e_{0}\left(j=\frac{5}{2}\right)$ $\approx 0.722 \mathrm{MeV}$ and $e_{0}\left(j=\frac{3}{2}\right)<0.722 \mathrm{MeV}$. The same trend is followed by the two points in the $1 s_{\frac{1}{2}}$-shell, while the points in the $1 p_{\frac{1}{2}}$ - and $2 s_{\frac{1}{2}}$-shells follow closely the trend in the preceding shells.

The quantity $e_{0}$ seems to depend on $T$. The effect is most pronounced in the region $A=50-60$ (above $Z=20$ ) where $e_{0}$ decreases slightly with increasing $T$. Considering all experimental information one must conclude, however, that the $T$-dependence of $e_{0}$ is relatively weak.

The slope $e_{0}$ changes at $N=50$ and at $Z=50$. In the region between $N=50$ and $Z=50$ a quadratic $A$-dependence is suggested by the experimental data. This would mean that $e_{0}$ is also a weak function of $A$.

The quantity $e_{1}$ is weakly $T$-dependent. Despite the limited data which is available small departures from an equal spacing between the lines for neighboring $T$ can be recognized.

The preceding analysis shows that nuclear structure effects can be seen for $T \leqq 1$ and $T>1$ in a plot of the quantity $A^{\frac{1}{3}} \Delta E_{\mathrm{Coul}}^{(\mathrm{red})}-(0.722 \mathrm{MeV}) A$ as a function of $A$. More experimental data are needed for $T>1$ to demonstrate the effects more clearly. Because of the still limited number of data which is available no effort was made to derive best numerical values for the parameters. Nevertheless, Fig. 3 can be used to predict within certain ranges of nuclei relatively accurate values for the Coulomb energy differences (Coulomb displacement energies) between isobaric analogue states.

After the completion of this work two preprints of publications on closely related subjects became available. LoNG et al. ${ }^{15}$ consider the quantity $\Delta E_{\text {Coul }}-\left(b_{1} Z / A^{\frac{1}{3}}+b_{2}\right)$ as a function of $Z / A^{\frac{1}{3}}$. HARCHOL et al. ${ }^{16}$ consider the same quantity as a function of $N$. The parameters $b_{1}$ and $b_{2}$ were obtained from least square fits to the data. The experimental data presented in these papers ${ }^{15,16}$ were included in the present analysis. Several of the papers originally quoted in ref. ${ }^{13}$ were eliminated and only those references were listed which are not part of any of the other compilations.

14 Thomas, R. G.: Phys. Rev. 81, 148 (1951); Phys. Rev. 88, 1109 (1952). Ehrman, J.B.: Phys. Rev. 81, 412 (1951). 
The semi-empirical equation

$$
\Delta E_{\mathrm{Coul}} \approx b_{1} \frac{\bar{Z}}{A^{\frac{1}{3}}}+b_{2}
$$

is often used ${ }^{6,10,15,16}$ to describe the gross structure of the experimental Coulomb energy differences. Considering the theoretical interpretation of the numerical coefficients in terms of an average effective electrostatic interaction $^{11}$ it appears more reasonable to use the equation

$$
\Delta E_{\mathrm{Coul}} \approx \frac{1}{A^{\frac{1}{3}}}\left(c_{1} \bar{Z}+c_{2}\right)
$$

instead. The factor $1 / A^{\frac{1}{3}}$ should apply to the term proportional to $\bar{Z}$ [other powers of $\bar{Z}$ are not possible; see Eq. (1)] and to the term independent of $\bar{Z}$ because both are related to expectation values of $1 / R$. Eq. (9) follows directly from Eq. (7) with the approximations $c_{1}=e_{1}=2 e_{0}=$ constant, $c_{2}=e_{2}-\frac{1}{2} e_{1}=$ constant, $e_{3}=0$. Neglecting any $N$-dependence of $A^{\frac{1}{3}} \Delta E_{\text {Coul }}$ a least square fit to all experimental data with $Z>20$ gives $c_{1}=(1.390 \pm 0.003) \mathrm{MeV}$ and $c_{2}=-(2.814 \pm 0.139) \mathrm{MeV}$. The standard error of the estimate, that is the root mean square of the deviations of $A^{\frac{1}{3}} \Delta E_{\text {Coul }}$ about the fitted curve $c_{1} \bar{Z}+c_{2}$, is about $250 \mathrm{keV}$. The standard error of $\Delta E_{\text {Coul }}$ is therefore of the order of $100 \mathrm{keV}$. A plot of the quantity $A^{\frac{1}{3}}\left\{\Delta E_{\text {Coul }}(\exp )-\Delta E_{\text {Coul }}\right.$ (calc) $\}$ as a function of $\bar{Z}$ (not shown) exhibits a systematic behavior which confirms that the fine structure effects of the Coulomb energies depend on $N$ and $Z$ or on $A$ and $T$.

The author acknowledges many stimulating discussions with K.T. HEchT.

15 Long, D.D., F. Richard, C.F. Moore, and J.D. Fox: To be published.

${ }^{16}$ Harchol, M., A. A. JAFFeE, J. Miron, I. UnNA, and J. Zionx: To be published. 